\title{
Studies of niobium thin film produced by energetic vacuum deposition
}

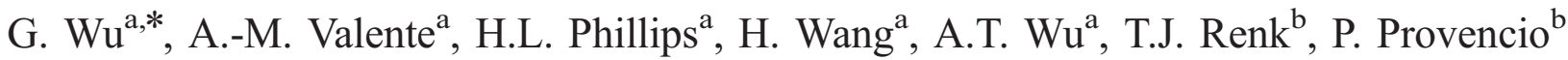 \\ ${ }^{a}$ Jefferson Lab, 12000 Jefferson Avenue, Newport News, VA 23606, USA \\ ${ }^{\mathrm{b}}$ Sandia National Laboratories, Albuquerque, NM 87185, USA
}

Received 12 May 2004; received in revised form 12 April 2005; accepted 28 April 2005

Available online 4 June 2005

\begin{abstract}
An energetic vacuum deposition system has been used to study deposition energy effects on the properties of niobium thin films on copper and sapphire substrates. The absence of a working gas avoids the gaseous inclusions commonly seen with sputtering deposition. A biased substrate holder controls the deposition energy. Transition temperature and residual resistivity ratio of the niobium thin films at several deposition energies are obtained together with surface morphology and crystal orientation measurements by atomic force microscope inspection, X-ray diffraction analysis and transmitted electron microscope (TEM) analysis. The results show that niobium thin films on a sapphire substrate exhibit the best cryogenic properties at a deposition energy of around $123 \mathrm{eV}$. The TEM analysis revealed that epitaxial growth of film was evident when the deposition energy reached $163 \mathrm{eV}$ for a sapphire substrate. Similarly, niobium thin films on copper substrates show that the film grows more oriented with higher deposition energy and the grain size reaches the scale of the film thickness at a deposition energy of around $153 \mathrm{eV}$.
\end{abstract}

(C) 2005 Elsevier B.V. All rights reserved.

Keywords: Niobium; Energetic condensation; Transmission electron microscopy; Electron cyclotron resonance

\section{Introduction}

Niobium thin film technology has been successfully used in several particle accelerators [1]. The commonly used magnetron sputtering technique to make accelerator components needs further improvements [2] for broader adoption in very high energy accelerator projects [3]. This paper describes thin film studies using an alternative coating technique, which has potential to be adopted in preparing niobium thin films inside radio frequency cavities used in superconducting particle accelerators.

Past experience with thin film growth has shown that higher surface adatom mobility [4] helps to suppress the columnar growth that has been commonly seen in magnetron sputtered niobium thin films on copper substrate. Very good results were reported when a niobium thin film was

\footnotetext{
* Corresponding author.

E-mail address: genfa@jlab.org (G. Wu).
}

magnetron sputtered on a heated sapphire substrate [5]. Since a copper substrate cannot support sufficiently elevated temperatures to achieve the needed mobility thermally, energized niobium has been used in biased magnetron sputtering [6]. Meanwhile, vacuum arc deposition [7,8] can yield a very high rate of niobium ions [9], which in turn helps to achieve greater surface adatom mobility.

An electron cyclotron resonance (ECR) $[10,11]$ plasma metal ion source is the ideal tool for direct niobium thin film deposition with the flexibility to adjust the niobium ion deposition energy [12]. Ultra-high vacuum and freedom from macroparticles are added advantages for this ion source.

For niobium thin films deposited at different energies, transition temperature and residual resistivity ratio (RRR) were measured. Film surfaces were inspected by atomic force microscopy (AFM), and crystal orientations were checked through X-ray diffraction. Cross sections of several films on sapphire and copper substrates have 


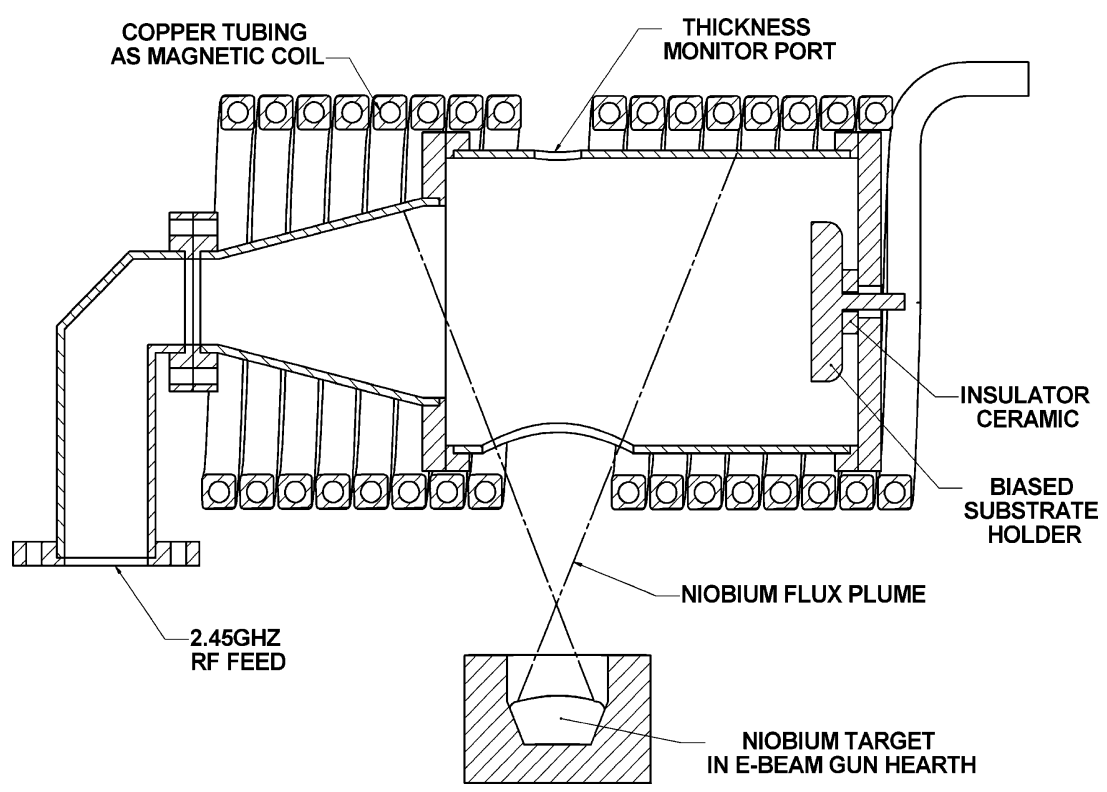

Fig. 1. Experimental apparatus.

been analyzed by transmission electron microscopy (TEM).

\section{Experimental details}

To generate niobium metal ions inside a vacuum, a thermionic electron gun is used to create a dense neutral niobium flux, and an ECR chamber is used to convert the passing neutral niobium vapors to niobium plasma as shown in Fig. 1. The ECR chamber is made of a copper cylinder that is inserted into the two slightly separated copper coils. The ECR chamber, copper coils and the electron gun sit in the vacuum chamber. When the cylinder is connected to the RF source, an electric field is established perpendicular to the magnetic field created by the copper coils. The niobium plasma is created when the electrons resonate with the RF field. Energetic

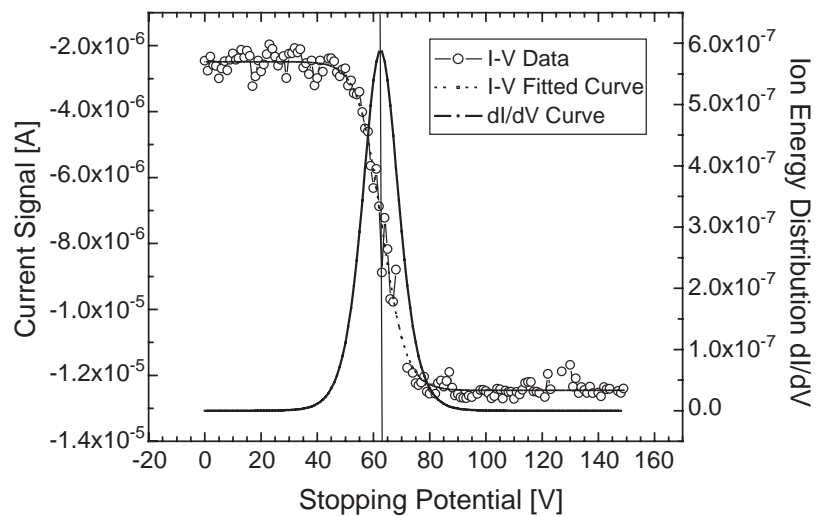

Fig. 2. The $\mathrm{I}-\mathrm{V}$ data obtained from an ion energy analyzer measuring niobium ions at the substrate without bias voltage. niobium ions are extracted to a biased substrate [13]. Deposition energy is simply controlled by the substrate bias voltage. A three-grid retarding field energy analyzer was used to measure the niobium ion current at the substrate location. When a stopping potential was applied to the analyzer center grid, an $\mathrm{I}-\mathrm{V}$ curve was obtained. Since the ECR plasma has low density, a single charge state is assumed for niobium ions. Thus, the energy distribution of the niobium ions can be obtained [14]. The result is shown in Fig. 2 when no bias voltage was applied. It shows that the niobium plasma has static potential of 63 $\mathrm{V}$. The energy spread for niobium ions at the substrate location is $20 \mathrm{eV}$. As a result, the ion energy at the substrate location is the energy gained by the sum of accelerating voltage of the bias voltage and the plasma static potential $(63 \mathrm{~V})$.

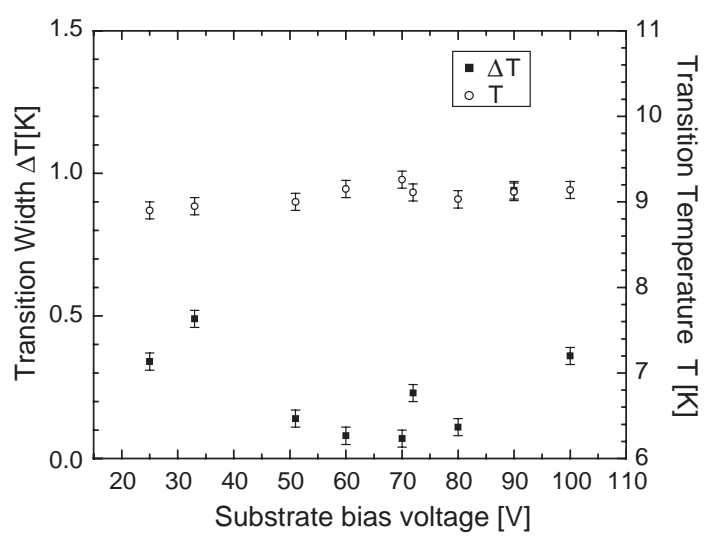

Fig. 3. Superconducting transition temperature and transition width of niobium films deposited on sapphire at various substrate bias voltages. Substrates were A-cut sapphire. 
For each thin film deposition, the chamber vacuum pressure was $3.0 \times 10^{-6} \mathrm{~Pa}$ before deposition, and $5.0 \times 10^{-5} \mathrm{~Pa}$ during deposition. The residual gas was mostly hydrogen. The actual vacuum pressure inside the ECR chamber was substantially lower, but was not directly measured. The deposition rate was $3-5 \AA / \mathrm{s}$ for the thin film samples described in this article. The current film thickness was limited to $300 \mathrm{~nm}$ due to the limited niobium volume in electron gun hearth. The substrates for each deposition included two sapphire and one copper disks. To insure a clean smooth substrate, at least $150 \mu \mathrm{m}$ of material was removed by electropolishing on the copper disk. For sapphire substrate, a thin layer of gold was sputtered onto a $2 \mathrm{~mm}$ edge in different deposition chamber before installed in the ECR chamber. This gold tab helped to reduce substrate charging during initial film growth and provided bias voltage to the otherwise insulated sapphire substrate.

Niobium films' superconducting transition was observed through the measurement of the films' ability to shield the magnetic field generated by an alternating current. A film was inserted between two coils without contact. One coil served as the driven coil, which generated a sinus magnetic field. The other coil picked up the alternating magnetic field and the strength of the signal was measured during superconducting transition. The transition temperature was determined at the middle of the pickup signal strength change. The transition width was defined as the temperature range where the pickup signal strength changed between $10 \%$ and $90 \%$ of the signal strength difference. During the four-point RRR measurement, a $100 \mathrm{~mA}$ direct current was applied to the niobium film through two contacting points; voltage drop was measured between another two contacting points between 10 and $300 \mathrm{~K}$. RRR number was obtained as the ratio of the voltages between 300 and $10 \mathrm{~K}$.

Crystallographic orientation of the niobium films was obtained through $\theta-2 \theta$ scan of X-ray diffraction. The

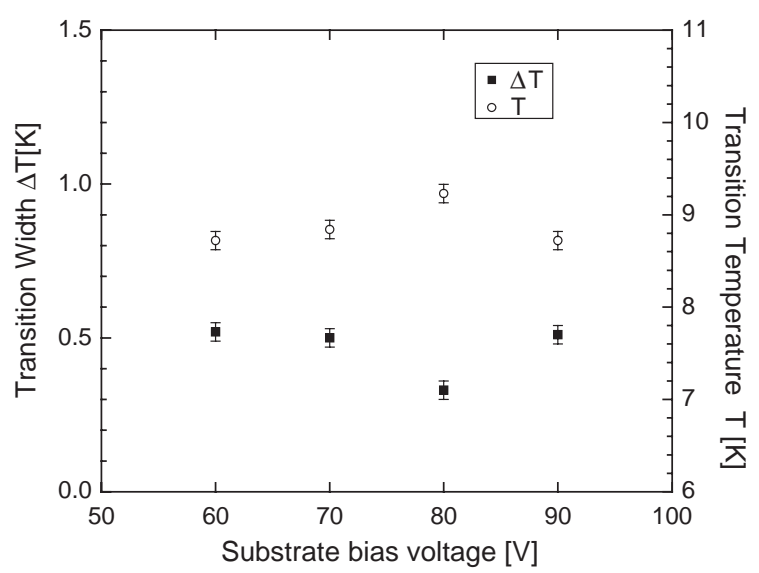

Fig. 4. Superconducting transition temperature and transition width of niobium films deposited on copper at various substrate bias voltages. Substrates were electropolished copper.

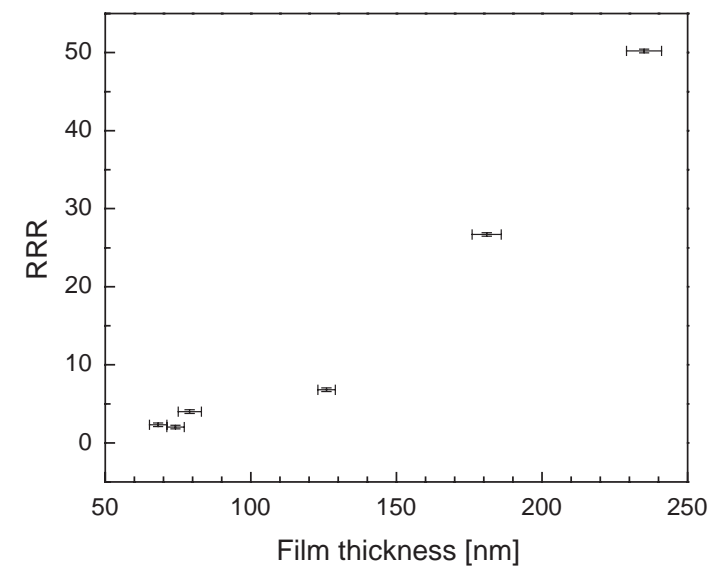

Fig. 5. RRR numbers for various thicknesses of niobium films deposited on sapphire.

surface morphology of niobium films was studied by AFM using the tapping mode. The cross section microtexture characterization of niobium films was performed by TEM.

\section{Transition temperature and residual resistivity ratio measurement}

The transition temperature is measured by the alternating current induction method. The transition temperature and transition width for films deposited at different bias voltages on sapphire and copper substrates are shown in Figs. 3 and 4. Commercially available high quality solid niobium yields $9.26 \mathrm{~K}$ with transition width at $0.04 \mathrm{~K}$ from the alternating current induction method.

The superconducting transition width can reflect certain aspects of thin film quality. One can argue that both the porous film structure and the high density of crystal defects can contribute to the density of pinning voids during the superconducting transition. Porous columnar growth can happen easily when the deposition energy is low and more often if the deposition angle is far away from $90^{\circ}$ $[15,16,17]$. On the other hand, higher deposition energy can cause the crystal to grow abnormally if the growth rate is relatively high [18]. Macroscopically, the transition width can reflect the overall crystallite quality. For niobium films based on sapphire, the sharpest transition is around

Table 1

Crystal orientation spread by X-ray diffraction, film thickness and RRR for films on sapphire substrate

\begin{tabular}{llcc}
\hline $\begin{array}{l}\text { Film bias voltage } \\
\text { number (negative) }\end{array}$ & $\begin{array}{l}\text { Full width of half } \\
\text { magnitude of angle of } \\
\text { X-ray diffraction signal }\end{array}$ & $\begin{array}{l}\text { Thickness } \\
(\mathrm{nm})\end{array}$ & RRR \\
\hline 2.0 & $10-15$ & 68.2 & 2.31 \\
25.0 & 3 & 126.0 & 6.8 \\
51.0 & $<1$ & 235.0 & 50.2 \\
72.0 & 2.5 & 181.0 & 26.7 \\
\hline
\end{tabular}




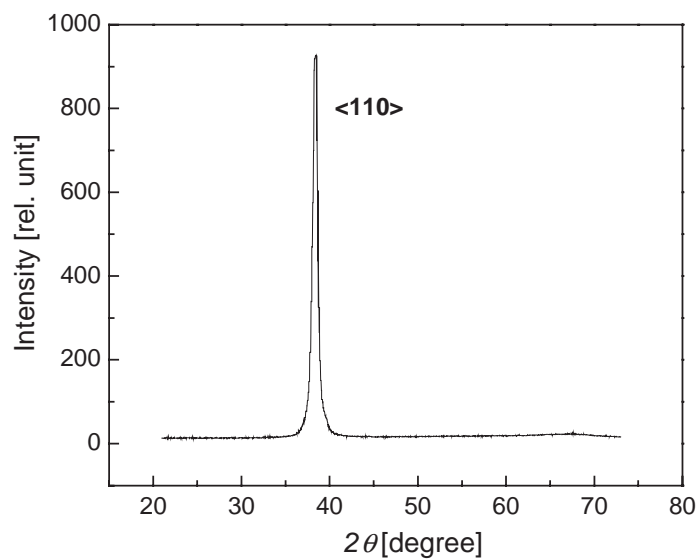

Fig. 6. X-ray diffraction spectra for a niobium film deposited on sapphire at $-51 \mathrm{~V}$ substrate bias.

$-60 \mathrm{~V}$ bias, which corresponds to a deposition energy around $123 \mathrm{eV}$.

In the case of niobium thin films on copper substrates, a brief inspection by optical microscope was used to ensure the copper surface quality. The transition temperature was fairly consistent, as shown in Fig. 4.

The second parameter to reflect the overall film quality is the RRR (residual resistivity ratio). The RRR value has been measured with a four-point resistive method and is defined as the ratio of resistivity at $300 \mathrm{~K}$ to the resistivity at $10 \mathrm{~K}$. RRR describes the metal purity in the sense of electron scattering due to alien atoms in the metal. In our case, RRR would reflect overall crystal quality, which includes porous structure, crystal defects, impurities and film thickness in the case of boundary scattering [19].

Measured RRR values increase with film thickness, as seen from Fig. 5. The lower RRR values are likely caused by two factors: the increased electron resistance by electron

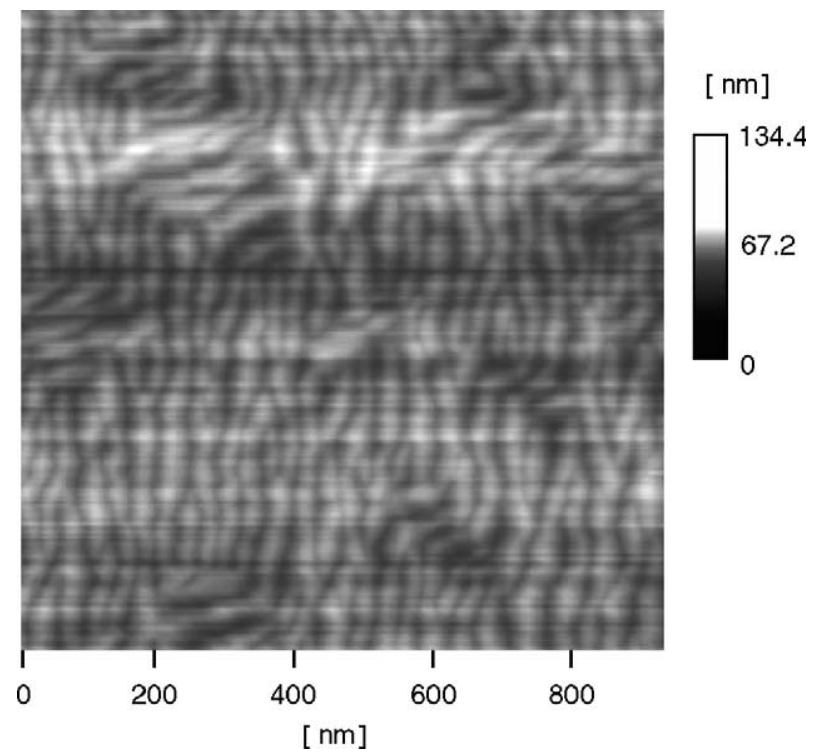

Fig. 7. AFM image of the surface of a niobium film deposited on sapphire at $-51 \mathrm{~V}$ substrate bias. boundary scattering in very thin films, and the increased density of crystal dislocation, as seen during TEM analysis. When film thickness is increased, the increased grain size becomes the dominating contribution for increased RRR, as shown in Table 1.

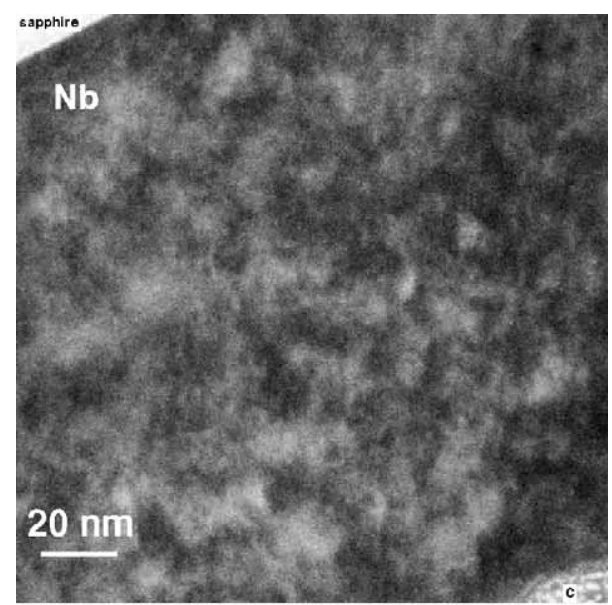

(a)

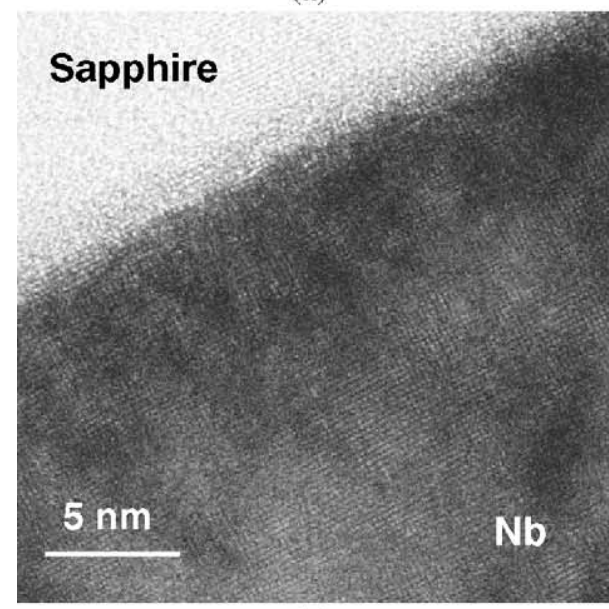

(b)

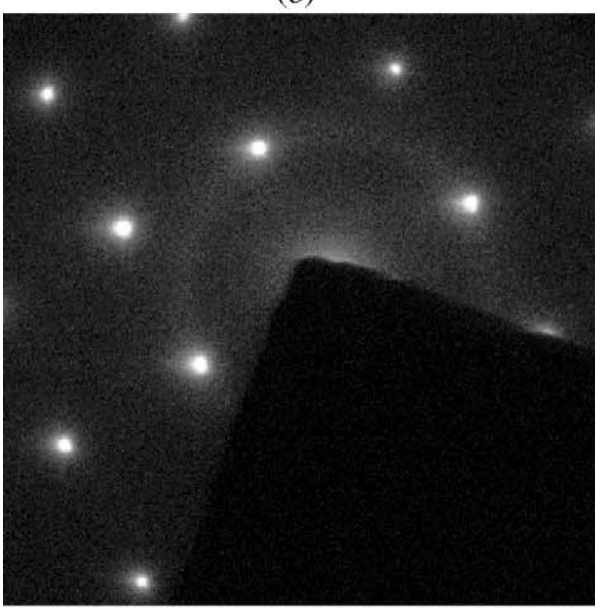

(c)

Fig. 8. Macrographs of a niobium film deposited on sapphire at $-100 \mathrm{~V}$ substrate bias: cross section TEM view (a), cross section view of niobium sapphire interface (b) and electron diffraction pattern showing $<110>$ crystal orientation (c). 


\section{X-ray diffraction and AFM surface inspection}

The crystal orientation of the films was determined by Xray diffraction. For both types of substrates, the film grows with a $<110>$ preferential orientation. An X-ray spectrum of niobium film deposited on sapphire at $-51 \mathrm{~V}$ is shown in Fig. 6 . The full width of half magnitude of the diffraction signal for the $\langle 110>$ crystal plane is shown in Table 1 . It shows that deposition energy changes the crystal orientation; particularly at $-51 \mathrm{~V}$, the niobium film structure is very oriented.

Fig. 7 shows the AFM measurement of a film deposited at $-51 \mathrm{~V}$ substrate bias. The surface morphology shows unusually long narrow grains compared with the round domeshaped grains seen in films at lower substrate bias voltage.

\section{Cross section TEM analysis for sapphire and copper based niobium films}

Some of the niobium films are currently being investigated by cross section TEM. A few interesting preliminary results have been obtained.
For films based on sapphire substrates, once the substrate bias reaches around $-51 \mathrm{~V}$, or deposition energy reaches $114 \mathrm{eV}$ and higher, most show good crystal orientation. For films deposited at $-100 \mathrm{~V}$ bias, the angle deviations are from crystal mismatch instead of large grain gaps. For that film, the interface between sapphire and niobium is very sharp, and shows epitaxial growth of $\mathrm{Nb}$ as shown in Fig. 8.

For films on copper substrates, the bias voltage was increased from -60 to $-90 \mathrm{~V}$ at an increment of $-10 \mathrm{~V}$. The cross section TEM pictures of film at $-60 \mathrm{~V}$ show polycrystalline film structure as shown in Fig. 9b. The grain size ranges from 5 to $20 \mathrm{~nm}$. When the bias voltage was increased, the size of domains or grains increased. Fig. 9c shows such a film deposited at $-90 \mathrm{~V}$ bias voltage with 100 $\mathrm{nm}$ grain size, which is at the same scale of film thickness. Fig. 9d shows a single grain boundary compared to the multiple grain grain boundaries in Fig. 9b. The -60 and $-90 \mathrm{~V}$ bias corresponds to deposition energies of 123 and $153 \mathrm{eV}$. This increased deposition energy is apparently responsible for the grain size variation for these films. This supposition was made more convincing by the measurement of electron diffraction pattern. The electron diffraction

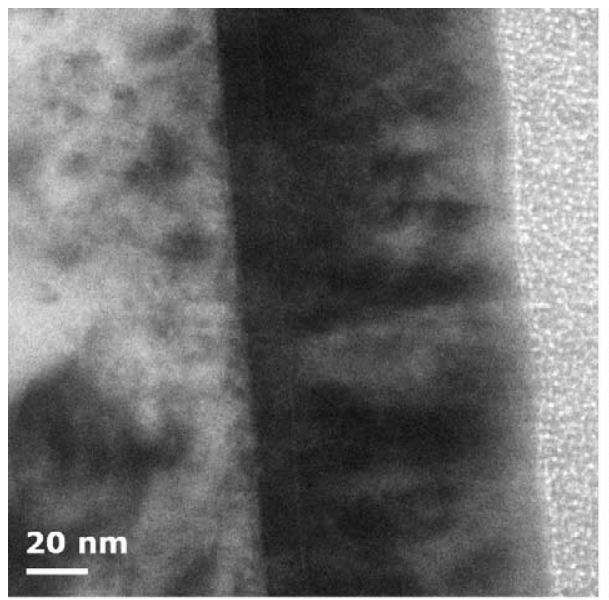

(a)

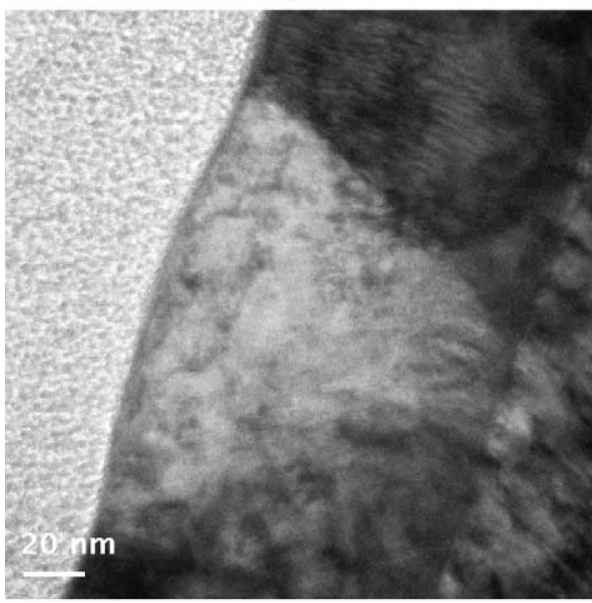

(c)

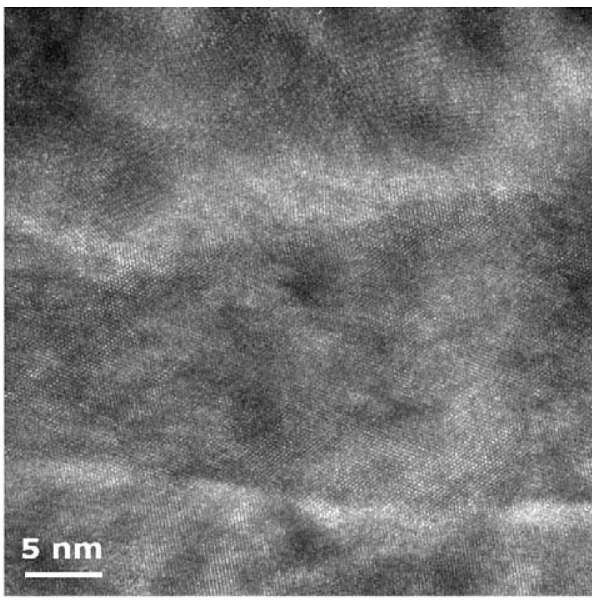

(b)

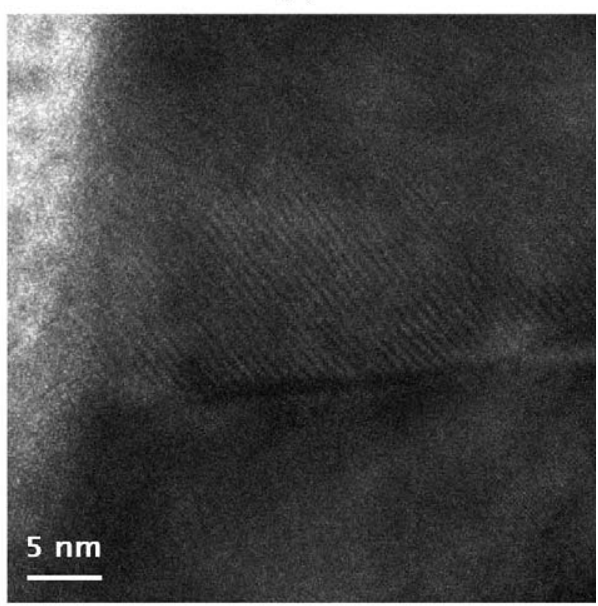

(d)

Fig. 9. Cross section micrographs of a niobium film deposited on copper at $-60 \mathrm{~V}(\mathrm{a}, \mathrm{b})$ and $-90 \mathrm{~V}(\mathrm{c}, \mathrm{d})$ substrate bias. 


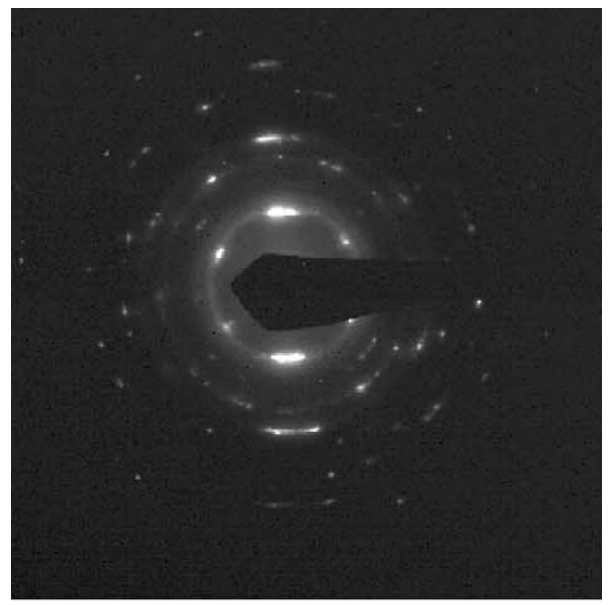

(a)

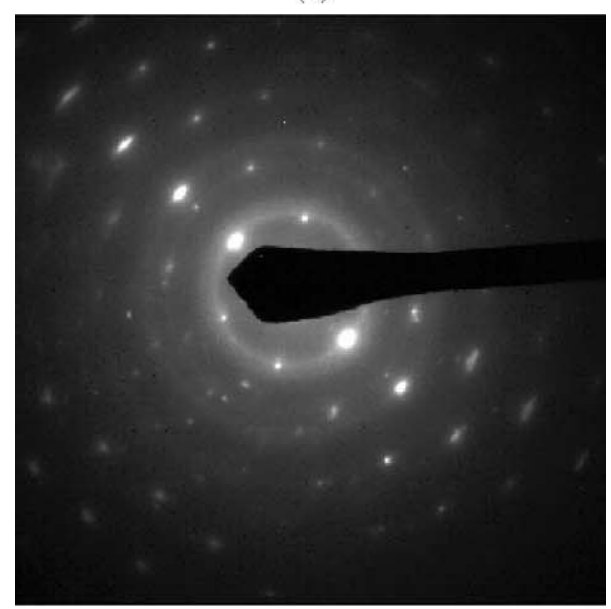

(b)

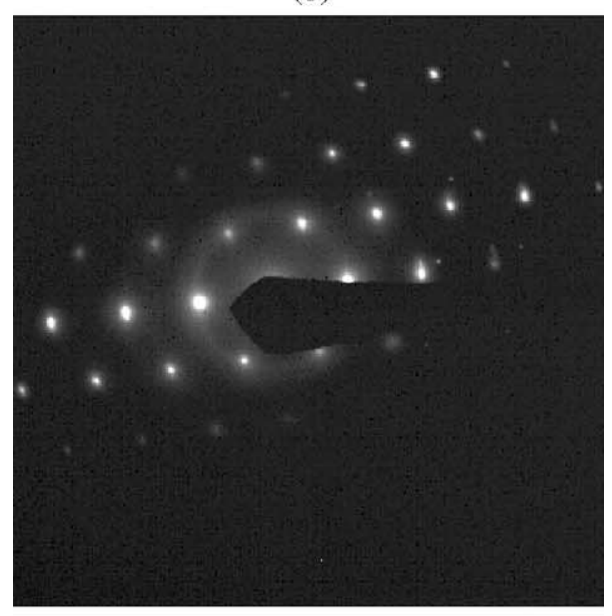

(c)

Fig. 10. Electron diffraction pattern of niobium film on copper deposited at respectively $-60 \mathrm{~V}(\mathrm{a}),-70 \mathrm{~V}$ (b) and $-90 \mathrm{~V}$ (c) substrate bias.

pattern for the -60 and $-90 \mathrm{~V}$ film is shown in Fig. 10a and c. Fig. $10 \mathrm{~b}$ is the electron diffraction pattern of $-70 \mathrm{~V}$ film. Fig. 10 shows a clear trend for more oriented film structure as the deposition energy was increased. Especially the $-90 \mathrm{~V}$ film shows a clean $<110>$ crystal plane. The surface flatness difference was apparently caused by the grain size difference as shown in Fig. 9a and c. Further TEM results will be given in a future publication.

\section{Conclusion}

Epitaxial growth of niobium on sapphire has been achieved for energetic deposition and this deposition process can help improve the film quality on copper substrates. Cryogenically, the process achieves a reasonably high RRR niobium thin film with excellent superconducting transition temperature and width. RRR appears to be more closely related to the film thickness than to the deposition energy. For sapphire substrates, the substrate bias voltage of $-60 \mathrm{Vs}$ is believed to be the preferred value based on the transition width, the crystal orientation spread, and the AFM results. That corresponds to a deposition energy around 123 eV. For a copper substrate, a similar film quality likely requires higher deposition energy, which needs further studies.

\section{Acknowledgements}

The authors are very grateful for technical support from Bret Lewis, Sam Morgan, Peter Kushnick, John Musson and many other Jefferson Lab staff members and also Brandt Robertson from Old Dominion University. The authors want to thank R.E. Crooks for his productive discussion and S. Corneliussen for his editorial help. The work was performed under DOE Contract \#DEAC0584ER40150. This work was also helped by L. Hand and made use of the Cornell Center for Materials Research Shared Experimental Facilities, supported through the National Science Foundation Research Science and Engineering Centers program (DMR-9632275).

\section{References}

[1] P. Brown, O. Brunner, A. Butterworth, E. Ciapala, H. Frischholz, G. Geschonke, E. Peschardt, J. Sladen, in: B. Rusnak (Ed.), 9th Workshop on RF SuperconductivitySanta Fe, U.S.A., November 15, 1999, Los Alamos National Laboratory, p. 1.

[2] V. Arbet-Engels, C. Benvenuti, S. Calatroni, P. Darriulat, M.A. Peck, A.-M. Valente, C.A. Van't Hof, Nucl. Instrum. Methods Phys. Res. A 463 (2001) 1.

[3] H. Padamsee, in: P. Lucas, S. Webber (Eds.), 2001 Particle Accelerator Conference, Chicago, U.S.A., June 18-22, 2001, IEEE, Inc., Piscataway, NJ, p. 468 (08855).

[4] J.A. Thornton, J. Vac. Sci. Technol 11 (1974) 666.

[5] L.N. Hand, J.P. Craig, J.A. Thompson, W.R. Frisken, in: D. Proch (Ed.), 11th Workshop on RF Superconductivity, Lübeck/Travemünde, Germany, September 8-12, 2003.

[6] K. Zhao, B. Zhang, J. Hao, D. Xie, L. Wang, Z. Shen, S. Quan, Y. Tang, K. Zhao, R.L. Geng, J. Chen, in: B. Rusnak (Ed.), 9th Workshop on RF Superconductivity, Santa Fe, U.S.A., November 1-5, 1999, Los Alamos National Laboratory, p. 70.

[7] Y. Igarashi, M. Kanayama, J. Appl. Phys. 57 (3) (1985) 849. 
[8] R. Russo, L. Catani, S. Tazzari, M. Cirillo, V. Merlo, J. Langner, in: S. Noguchi (Ed.), 10th Workshop on RF Super-conductivity, Tsukuba, Japan, September 6-11, 2001, KEK, Ibaraki, Japan, p. 44.

[9] I.G. Brown, J.E. Galvin, IEEE Trans. Plasma Sci 17 (1989) 679.

[10] W.M. Holber, J.S. Logan, H.J. Grabarz, J.T.C. Yeh, J.B.O. Caughman, A. Sugerman, F.E. Turene, J. Vac. Sci. Technol., A, Vac. Surf. Films 11 (1993) 2903.

[11] S.M. Rossnagel, J. Hopwood, J. Vac. Sci. Technol. B 12 (1994) 449.

[12] G. Wu, H.L. Phillips, R.M. Sundelin, J. Vac. Sci. Technol., A, Vac. Surf. Films 21 (4) (2003) 842.

[13] G. Wu, H.L. Phillips, R.M. Sundelin, T. Goodman, in: S. Noguchi (Ed.), 10th Workshop on RF Super-conductivity, Tsukuba, Japan, September 6-11, 2001, KEK, Ibaraki, Japan, p. 259
[14] C. Bohm, J. Perrin, Rev. Sci. Instrum. 64 (1993) 31.

[15] D.J. Srolovitz, A. Mazor, B.G. Bukiet, J. Vac. Sci. Technol., A, Vac. Surf. Films 6 (4) (1988) 2371.

[16] D. Vick, L.J. Friedricha, S.K. Dewa, M.J. Bretta, K. Robbiea, M. Setoa, T. Smy, Thin Solid Films 339 (1999) 88.

[17] D. Tonini, C. Greggio, G. Keppel, F. Laviano, M. Musiani, G. Torzo, V. Palmieri, in: D. Proch (Ed.), 11th Workshop on RF Superconductivity, Lübeck/Travemünde, Germany, September 8-12, 2003.

[18] J.M. Pomeroy, J. Jacobsen, C.C. Hill, B.H. Cooper, J.P. Sethna, Phys Rev. B 66 (2002) 235412.

[19] H.M. Rosenburg, Low Temperature Solid State Physics, Oxford University Press, London, 1963. 